\title{
Rietveld and pair distribution function study of Hägg carbide using synchrotron X-ray diffraction
}

\author{
Hester Esna du Plessis, ${ }^{a^{*}}$ J.P.R. de Villiers, ${ }^{b}$ G.J. Kruger, ${ }^{c}$ A. Steuwer ${ }^{d}$ \\ and M. Brunelli ${ }^{\mathrm{e}}$
}

${ }^{a}$ Sasol Technology R\&D, South Africa, ${ }^{b}$ University of Pretoria, South Africa, 'University of Johannesburg, South Africa, ${ }^{d} E S S$ Scandinavia, University of Lund, Stora Algatan 4, 22350 Lund, Sweden and NMMU, Port Elizabeth 6031, South Africa and ${ }^{\mathrm{e}}$ ILL Institut Laue-Langevin, BP 156, 38042 Grenoble cedex 9, France.

E-mail: axel.steuwer@esss.se

Synopsis The atomic configuration of the pseudo-monoclinic structure of Hägg carbide ( $\chi$ $\mathrm{Fe}_{5} \mathrm{C}_{2}$ ) was confirmed by a real-space modelling of the pair distribution function (PDF) and comparison with results from high-resolution synchrotron X-ray powder diffraction. The PDF of $\chi-\mathrm{Fe}_{5} \mathrm{C}_{2}$ compares well with that of a pseudo-monoclinic phase of space group P $\overline{1}$ ( $\mathrm{a}=$ 11.5661(6) $\AA, \mathrm{b}=4.5709(1) \AA, \mathrm{c}=5.0611(2) \AA, \alpha=89.990(5)^{\circ}, \beta=97.753(4){ }^{\circ}, \gamma=$ $\left.90.195(4)^{\circ}\right)$, where the carbon atoms are located in distorted prismatic trigonal and octahedral sites. The distortion is probably due to the mobility of carbon in the structure.

Abstract Fischer-Tropsch (FT) synthesis is an important process to manufacture hydrocarbons and oxygenated hydrocarbons from mixtures of carbon monoxide and hydrogen (syngas). The reduced iron catalyst reacts with carbon monoxide and hydrogen to form bulk $\mathrm{Fe}_{5} \mathrm{C}_{2}$ Hägg carbide $(\chi-\mathrm{HC})$ during FT synthesis. Arguably, $\chi$-HC is the predominant catalyst phase present in the working iron catalyst. Deactivation of the working catalyst can be due to oxidation of $\chi$-HC to iron oxide, a step wise decarburization to cementite $\left(\theta-\mathrm{Fe}_{3} \mathrm{C}\right)$, carbon formation or sintering with accompanying loss of catalytic performance. It is therefore critical to determine the precise crystal structure of $\chi$-HC for the understanding of the synthesis process and for comparison with the first-principles ab-initio modelling. Here we report on the results of high-resolution synchrotron X-ray powder diffraction data. The atomic arrangement of $\chi-\mathrm{HC}$ was confirmed by Rietveld refinement and subsequent real-space modeling of the pair distribution function (PDF) obtained from direct Fourier transformation. The Rietveld and PDF results of $\chi$-HC correspond well to that of a pseudo-monoclinic phase of space group $\mathrm{P} \overline{1}\left(\mathrm{a}=11.5661(6) \AA, \mathrm{b}=4.5709(1) \AA, \mathrm{c}=5.0611(2) \AA, \alpha=89.990(5)^{\circ}, \beta=\right.$ 97.753(4) $\left.{ }^{\circ}, \gamma=90.195(4)^{\circ}\right)$ ), where the iron atoms are located in three distorted prismatic trigonal and one octahedral arrangement around the central carbon atoms. The iron atoms are 
distorted from the prismatic trigonal arrangement in the monoclinic structure by the change in carbon atom location in the structure.

\section{Keywords: Hägg carbide; Fischer-Tropsch synthesis; crystal structure; PDF}

\section{Introduction}

Recent fluctuating oil prices and increasing environmental concerns have stimulated renewed interest in the production of synthetic hydrocarbons (fuels) by Fischer-Tropsch synthesis (FTS) using biomass, natural gas or coal as starting material (Dry, 1990; Riedel \& Schulz, 2003). The FTS involves a catalyst, and one of the most commonly used commercial catalyst is Fe-based, starting from oxides with small amounts of promoter substances such as $\mathrm{Cu}, \mathrm{K}, \mathrm{SiO}_{2}$ added to improve performance (Herranz et al, 2006; Niemantsverdriet, 2008). The Fe-catalysts requires an activation pre-treatment (using $\mathrm{CO}$, syngas, hydrogen) and to increase the efficiency, nano-scale oxide powders with high surface to bulk ratios are being used (Sarkar et al, 2007). During activation the iron oxides evolve, resulting in the formation of metallic iron and a range of carbides depending on temperature, pressure of carbon containing gases, concentration, time of exposure etc. Subsequent annealing results in a diffusion-determined de-carburization process to form stable cementite. Cementite and other iron carbide phases have also been observed to form via lattice-invariant deformation after carbon ion implantation into thin iron layers (Königer, 2006). Experimental evidence suggests that the activity (and hence performance) of the Fe-based catalyst is critically dependent on the presence and type of carbides on the surface (Herranz et al, 2006). There is a range of meta-stable and stable iron-carbides, and the differences in crystal structure are relatively subtle but distinct, and can be confirmed with the aid of electron diffraction (Hirotsu \& Nagakura, 1972; du Plessis, de Villiers \& Kruger, 2007). In general, the iron-carbides can be divided into carbides with carbon atoms on octahedral interstitial sites (O-carbides) such as $\varepsilon$ -

$\mathrm{Fe}_{2} \mathrm{C}, \varepsilon-\mathrm{Fe}_{2.2} \mathrm{C}$ and $\mathrm{Fe}_{\mathrm{x}} \mathrm{C}$, and carbides with carbon atoms on trigonal prismatic interstices such as $\eta-\mathrm{Fe}_{2} \mathrm{C}$ and $\chi-\mathrm{Fe}_{5} \mathrm{C}_{2}$ (Hägg) and the (stable) cementite phase $\theta-\mathrm{Fe}_{3} \mathrm{C}$. The exact nature of the carbonaceous species on the surface and in the bulk over the catalyst has been the subject of intense investigations over recent years (Herranz et al, 2006; Steynberg, van den Berg \& Janse van Rensburg, 2008; Faraoun et al, 2006; Mansker, 1999; Pérez-Alfonso et $a l$, 2007), but experimental evidence points at the Hägg carbide being the prevalent phase at 'steady-state' FTS conditions and principally responsible for FTS activity. During FischerTropsch synthesis the partial pressure of syngas is kept constant, promoters are added to aid the dissociation of carbon and oxygen on the catalyst surface and the de-carburization process of Hägg carbide to cementite is thus limited. 
The oxidation of iron and iron carbides to magnetite is a postulated deactivation mechanism of the catalyst, due to water-gas-shift reaction taking place (Sarkar et al, 2007; Mansker, 1999). Molecular dynamic simulation studies of the interaction of carbon with selfinterstitial clusters in $\alpha$-iron indicated the octahedral site as the most stable one for the carbon interstitial and the tetrahedral site as the saddle-point for carbon jump from one octahedral site to another (Tapasa et al, 2007). It was shown that the iron lattice volume expands due to a carbon atom addition to self-interstitial atom clusters. At $300 \mathrm{~K}$ a cluster of seven selfinterstitial-atoms with one or two carbon atoms was found to be essentially immobilized. The cluster co-migrated with carbon atoms at 600 and $900 \mathrm{~K}$. Dissociation of carbon from the iron cluster at $1200 \mathrm{~K}$ was reported.

The self-diffusion coefficients of carbon at $773 \mathrm{~K}$ were determined in both cementite and Hägg carbide under high carburising atmospheres (Scheider et al, 2007). Metal dusting was applied to limit graphite deposition. The mechanism of carbon diffusion is not known and the carbon self-diffusion coefficient is dependent on the chemical potential difference between the surfaces and interfaces of phases containing different stoichiometric abundances of carbon.

The accurate structure determination of the carbides during FTS conditions is challenging, and further complicated by the small crystallite size of the powders (Herranz et al, 2006; Niemantsverdriet, 1980; du Plessis, de Villiers \& Kruger, 2007, Haegg, 1931, Retief, 1999; Senateur, 1962). The meta-stable carbides are very sensitive to exposure to air/water and changes in temperature, with may result in rapid deactivation of the working catalyst due to oxidation of $\chi$ - $\mathrm{HC}$ to iron oxide, a step wise decarburization to cementite $\left(\theta-\mathrm{Fe}_{3} \mathrm{C}\right)$, carbon formation and/or sintering. However, the fundamental understanding of the catalysis process and the focussed development towards more efficient catalyst using molecular dynamics tools requires a better understanding of the precise interaction between carbon and iron, and the precise carbide structure and distribution during FTS.

Recently, the crystal structure was re-determined by laboratory powder X-ray diffraction and selected area electron diffraction (du Plessis, de Villiers \& Kruger, 2007). The agreement of structure factors between the calculated and experimental powder patterns was significantly improved when using the triclinic structure instead of the monoclinic structure as previously reported by Senateur (Senateur, 1962) of $\chi$-HC. Small, but statistically significant differences (Steynberg, van den Berg, Janse van Rensburg, 2008) in the refinement parameters when using monoclinic and pseudo-monoclinic structures of $\chi$-HC exposed the need for higher resolution synchrotron X-ray diffraction data. The pseudo-monoclinic crystal structure was re-determined from selected area electron diffraction and powder XRD by using simulated annealing, a direct space structure determination algorithm in Topas (Coelho, 
2000). It had to be determined whether the bulk structure of $\chi$-HC extended to the surface of the particles, since this will be the interface available for FTS. Quantitative phase analysis using total-scattering analysis (PDF) was used to determine whether amorphous surface species exist on the $\chi$-HC crystallites. In this manuscript we report on the results of the synchrotron X-ray powder diffraction experiments undertaken on beam line ID31 at the ESRF, Grenoble. In addition to conventional high-resolution Bragg scattering, the available range in diffraction angles (or rather Fourier space) further allowed the total-scattering analysis of the diffractogram using the PDF approach (Qiu, Thompson \& Billinge, 2004).

\section{Experimental}

A synthetic powder sample with particle sizes between 5 to $40 \mu \mathrm{m}$ (Figure 1) containing mainly $\chi$-HC was prepared by carburization of iron with carbon monoxide at $330{ }^{\circ} \mathrm{C}$ for 6 hours. The individual grains are of the order of tens of nanometres (as determined by Rietveld refinement) and not visible in Figure 1.

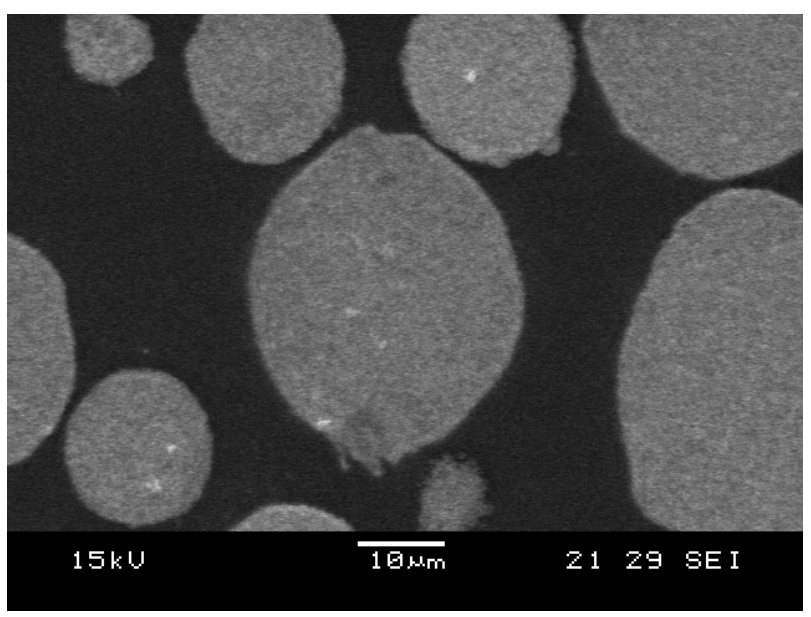

Figure 1 SEM back-scattered image of Hägg carbide sample.

The sample contained no catalysis promoters and can be considered to be a model FischerTropsch catalyst. $\chi$-HC was passivated at room temperature by exposing the sample to $0.5 \%$ oxygen in helium for $2 \mathrm{~h}$ to prevent oxidation during unloading of the iron carbide. The unloaded powder was packed in a $0.50 \mathrm{~mm}$ capillary. The diffraction experiment was undertaken on beam line ID31 at the ESRF, Grenoble. The wavelength was set to $0.3947 \AA$, equivalent of $31.4 \mathrm{keV}$. The diffractogram was collected to a maximum angle of $45^{\circ} 2 \theta$ (step size $\left.0.0085^{\circ} 2 \theta\right)$ to obtain the resolution required for accurate structure determination and refinement, as well as the PDF analysis. Longer counting times were used at higher Q-space values. The Q-space probed was thus 1.11 to $12.18 \AA^{-1}$ ). A reference standard, $\mathrm{LaB}_{6}$, was measured for characterising the experimental configuration. The software package Topas 4 was used for Rietveld refinement. The pair distribution function (PDF) was determined by 
direct Fourier transform of the total X-ray scattering data using the software PDFGetX2 (Qiu, Thompson \& Billinge, 2004).

\section{Results}

$\underline{\text { Results from Rietveld analysis: }}$

The basic crystal structure of $\chi$-Hägg was re-determined by laboratory powder X-ray diffraction and selected area electron diffraction (du Plessis, de Villiers \& Kruger, 2007) and the current measurements on ID31 follow essentially the same approach and are therefore reported here in slightly abbreviated fashion. The fundamental parameter approach was used to deconvolute instrumental and sample broadening observed in the diffractogram. The instrumental broadening was determined by refinement of a reference $\mathrm{LaB}_{6}$ diffractogram, using instrumental broadening parameters as available in Topas4. The instrumental broadening parameters were then fixed during Rietveld refinement of the $\chi-\mathrm{HC}$ diffractogram. Line broadening observed for the individual crystalline phases were then modeled using the appropriate phase-dependent parameters available in Topas4. During Rietveld refinement of the final $\chi$-Hägg carbide structure using the pseudo-monoclinic space group $\mathrm{P} \overline{1}$ the atomic displacement parameters of all the iron atoms in the phases were restrained as a common isotropic parameter which refined to a value of $u=0.25(2) \AA^{2}$ and the common isotropic parameter for the carbon atoms in the iron carbide phases refined to $\mathrm{u}=$ 0.01(3) $\AA^{2}$. The isotropic atomic displacement parameter of the carbon atoms in $\chi$-Hägg carbide was restrained with a minimum limit of $0.01 \AA^{2}$, as this parameter diverge to negative values during an unconstrained Rietveld refinement. All atomic sites in the crystalline phases were fully occupied $(\mathrm{SOF}=1)$. The published structures of $\chi$-HC (du Plessis, de Villiers $\&$ Kruger, 2007), $\theta-\mathrm{Fe}_{3} \mathrm{C}$ (Meinhardt $\&$ Krisement, 1962) and $\alpha-\mathrm{Fe}$ (Swanson et al, 1955) were used. The sample contained amorphous carbon formed during preparation of the sample and this was modelled as a single broad peak and therefore excluded from the quantitative phase analysis. The crystalline size broadening contribution to the $\chi$-HC reflections was modelled with a Lorentzian function and the volume weighted mean column height $\mathrm{L}_{\mathrm{V}_{\mathrm{ol}}}$ (Snyder, Bunge, Fiala, 1999) was found to be 36(1) nm from the Rietveld refinement. Similarly, the strain-broadening contribution was modelled using a Voigt function and the apparent strainboradening value was found to be $\varepsilon_{0}=0.075(2)$. The Rietveld refinement approach was as follows: the instrumental broadening contribution was determined from a refinement of the $\mathrm{LaB}_{6}$ standard. This instrument parameter file was then used for the Rietveld refinement of the $\chi$-HC sample. After determination of the zero error $(0.004(1) \AA)$, this value was fixed and the lattice parameters and relative atomic coordinates of $\chi-\mathrm{HC}$ were refined. The lattice parameters and relative atomic coordinates of $\theta-\mathrm{Fe}_{3} \mathrm{C}$ and $\alpha-\mathrm{Fe}$ were fixed to the published 
values for the initial Rietveld refinement. Upon obtaining an improved fit of $\chi$-HC to the experimental diffractogram, the $\theta-\mathrm{Fe}_{3} \mathrm{C}$ and $\alpha-\mathrm{Fe}$ lattice parameters and average crystallite sizes were refined. The improved values were then fixed and the final step was to refine the lattice parameter, relative atomic coordinates and average crystallite size of $\chi$-HC. The agreement values (Young, 1995) were: $\mathrm{R}_{\mathrm{wp}}$ of $9.25 \%, \mathrm{~S}=2.9 \%$ and $\mathrm{R}_{\text {Bragg }}$ of $4.0 \%$, as compared to a $\mathrm{R}_{\mathrm{wp}}$ of $12.7 \%, \mathrm{~S}=4.0 \%$ and $\mathrm{R}_{\mathrm{Bragg}}$ of $6.6 \%$ when refining the same synchrotron diffraction data using the structure as published by Retief (Retief, 1999). The experimental and theoretical diffractograms of $\chi-\mathrm{HC}$ can be seen in Figure 2, together with a visualisation of the unit cell.

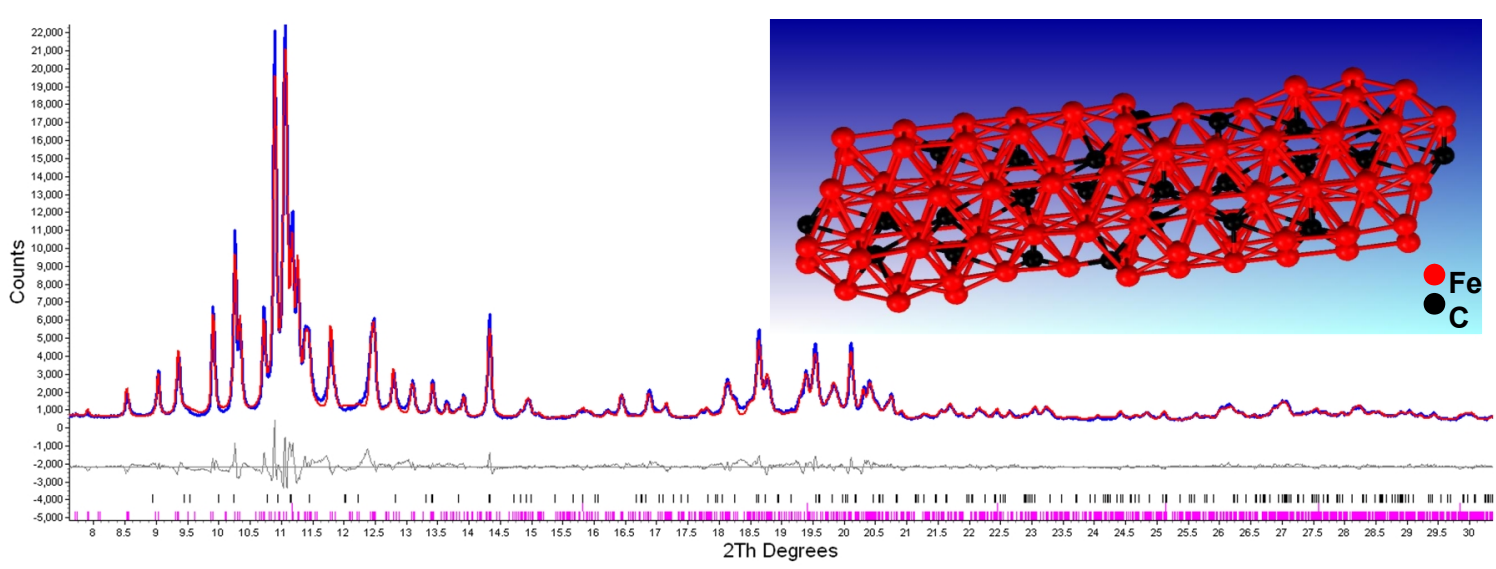

Figure 2 The experimental (blue) and theoretical (red) diffractograms of $\boldsymbol{\chi}-\mathbf{H C}, \theta-\mathrm{Fe}_{3} \mathrm{C}$ and $\alpha-\mathrm{Fe}$ $\left({ }^{\circ} 2 \theta, \lambda=0.39466 \AA\right)$. The inset shows the refined crystal structure, see also Figure 5.

\section{Results from PDF analysis:}

Unlike most conventional X-ray diffraction data, the diffractograms collected on ID31 lend themselves to analysis via pair distribution function (PDF) approach. The PDF (G(r)) for $\chi$ $\mathrm{HC}, \theta-\mathrm{Fe}_{3} \mathrm{C}$ and $\alpha$-Fe were calculated and refined using PDFGui software (Farrow et al, 2007). The fit between the experimental and theoretical pair distribution functions is shown in Figure 3. The lattice parameters, relative abundances of the phases and relative atomic coordinates and atomic displacement parameters thereof were refined for both the monoclinic and pseudo-monoclinic structures of $\chi$-HC. Since the bulk crystalline phases were identified and quantified during the Rietveld refinement analysis, these phases were used as starting models for the PDF analysis. The relative abundances and atomic coordinates of all the phases $\left(\chi-\mathrm{HC}, \theta-\mathrm{Fe}_{3} \mathrm{C}, \alpha-\mathrm{Fe}\right)$ were refined as well as the isotropic atomic displacement parameters. A spherical nano-particle shape was assumed and found to be sufficient. The peaks visible on the experimental PDF do not have an increased broadening at higher $\mathrm{r}$ values, indicating that there is no stacking disorder visible up to $20 \AA$. The PDF does not display a typical nano-crystalline decrease of peak intensities and this result correlates with 
the large average crystallite size obtained from Rietveld refinement, i.e. $36(1) \mathrm{nm}$. An $\mathrm{R}_{\mathrm{wp}}$ value of $12.9 \%$ and reduced $\chi^{2}$ value of $15.4 \%$ was obtained when fitting the experimental and theoretical PDFs when using the pseudo-monoclinic crystal structure of $\chi-\mathrm{HC}$, indicating that the bulk phases extended to the surface of the particles. The inter-atomic distances in the range of $1.8-2.2 \AA$ are assigned to iron to carbon, mainly from $\chi$-HC and the peak intensity and width matches well, indicating that all interatomic distances of $1.8-2.2 \AA$ in the sample are explained by the phases included in the PDF refinement. The larger interatomic distances can be assigned to iron-iron, iron-carbon and carbon-carbon interactions.

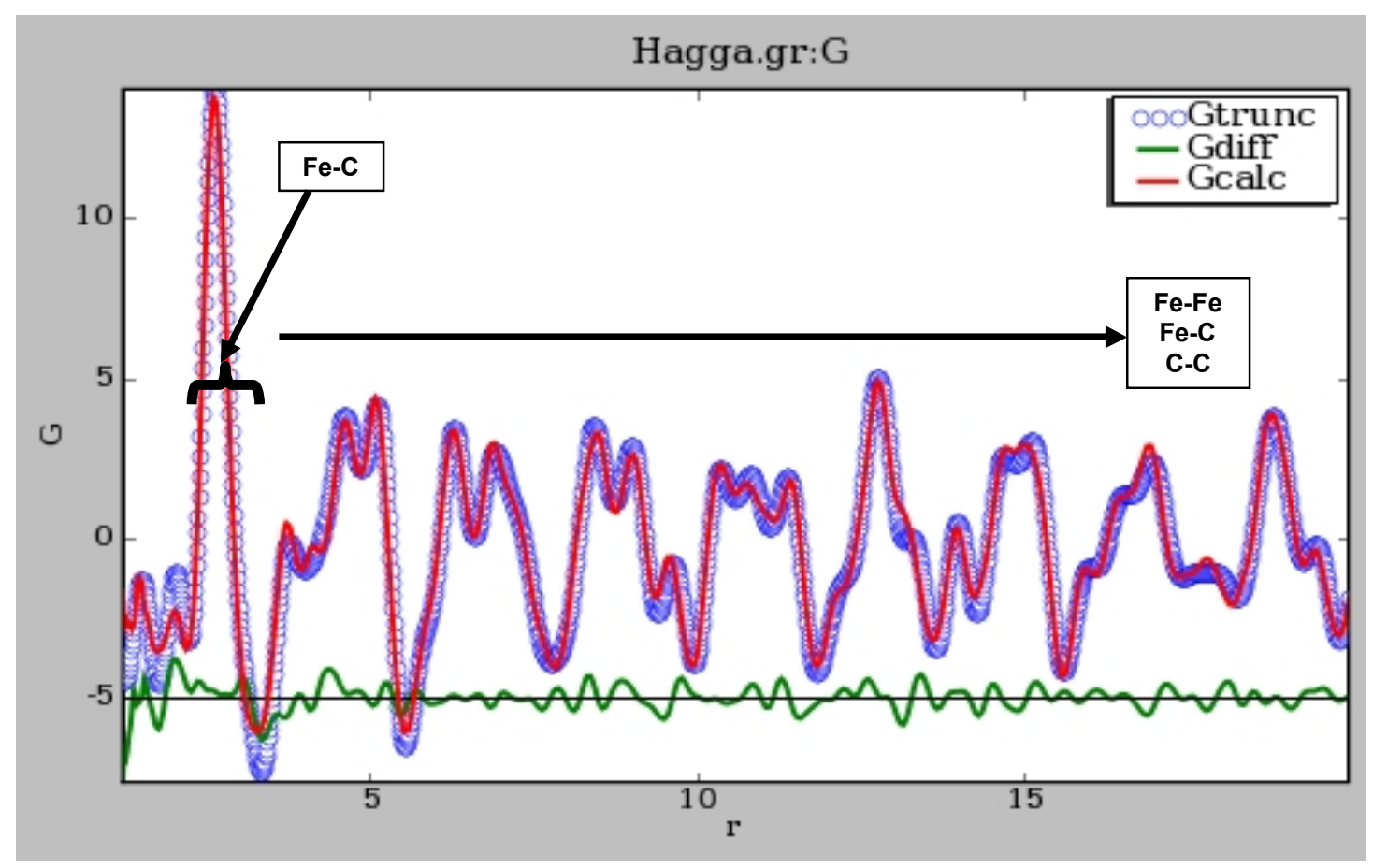

Figure 3 The PDF G(r) for $\chi-\mathrm{HC}, \theta-\mathrm{Fe}_{3} \mathrm{C}$ and $\alpha-\mathrm{Fe}$ (blue) with the refined fit of the model (red) and the difference pattern (green).

The presence of 1-2\%weight $\alpha$-iron (reactant) was detected in the synchrotron X-ray diffractogram when doing Rietveld refinement, which was not visible using laboratory X-ray data, probably due to the small crystallite size $(44 \mathrm{~nm}$, from Rietveld refinement) and peak overlap of the (011) reflection of $\alpha$-iron with the $\chi$-HC (121) reflection. The $\alpha$-iron phase was included during a trial Rietveld refinement of the laboratory X-ray data, but refined to zero. The (011) reflection of $\alpha$-iron in the synchrotron X-ray diffractogram is indicated with a red arrow in Figure 4. 


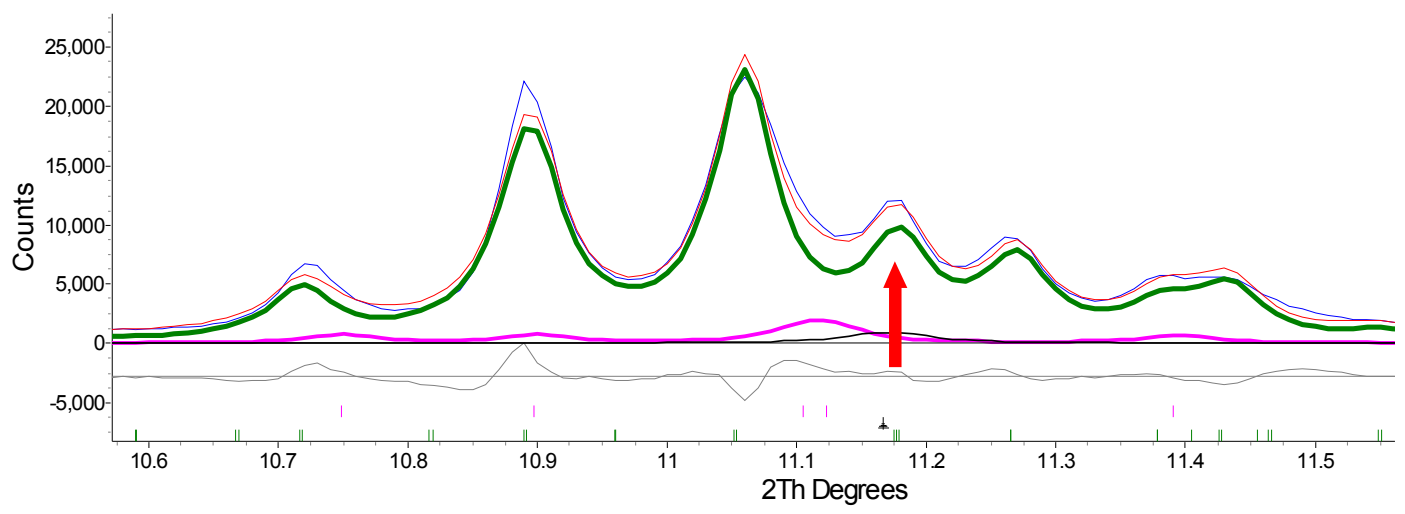

Figure 4 The experimental (blue) and theoretical (red) diffractograms and contributions of $\chi$-HC (green), $\theta-\mathrm{Fe}_{3} \mathrm{C}$ (pink) and $\alpha$ - $\mathrm{Fe}$ (black) $\left({ }^{\circ} 2 \theta, \lambda=0.39466 \AA\right.$ ).

However, using the PDF approach (Farrow et al, 2007; Qiu, Thompson \& Billinge, 2004) it was possible to refine the nano-crystalline structure of $\chi-\mathrm{HC}$, which confirmed the triclinic structure to be prevalent in the synthetically prepared $\chi$-HC. The lattice parameters, relative abundances, atomic displacement parameters and relative atomic coordinates of $\chi$-HC were optimized during the PDF refinements. A comparison of the relative abundances of the crystalline phases obtained from the Rietveld refinements (using the $\mathrm{C} 2 / \mathrm{c}$ and $\mathrm{P} \overline{1}$ crystal structures of $\chi-\mathrm{HC}$ ) and the agreement values are listed in Table 1:

Table 1 Relative abundances of phases

\begin{tabular}{|c|c|c|c|c|c|}
\hline & $\begin{array}{l}\text { Rietveld } \\
\mathrm{C} 2 / \mathrm{c}\end{array}$ & $\begin{array}{l}\text { Rietveld } \\
\mathrm{P}^{-}\end{array}$ & $\begin{array}{l}\mathrm{PDF} \\
\mathrm{C} 2 / \mathrm{c}\end{array}$ & $\begin{array}{l}\text { PDF } \\
\text { Pì }\end{array}$ & $\begin{array}{l}\text { Mössbauer } \\
\text { spectroscopy }\end{array}$ \\
\hline Relative abundances ( $\%$ weight $) *$ & & & & & \\
\hline$\chi-\mathrm{HC}$ & $80(1)$ & $84(1)$ & $93(1)$ & $90(3)$ & $85(1)$ \\
\hline$\theta-\mathrm{Fe}_{3} \mathrm{C}$ & $17(1)$ & $15(1)$ & $5(1)$ & $8(3)$ & $15(1)$ \\
\hline$\alpha-\mathrm{Fe}$ & $2(0.3)$ & $1(0.1)$ & $2(0.2)$ & $2(1)$ & - \\
\hline $\mathrm{R}_{\mathrm{wp}}(\%)$ & 12.74 & 9.01 & 15.5 & 12.9 & \\
\hline $\mathrm{R}_{\text {Bragg }}(\chi-\mathrm{HC})(\%)$ & 6.59 & 4.0 & & - & \\
\hline S (\%) & 4.0 & 2.9 & 21.5 & 15.4 & \\
\hline
\end{tabular}

* Results normalized to iron and iron carbides

It is clear from Table 1 that the crystal structure of $\chi-\mathrm{HC}$ is indeed $\mathrm{P} \overline{1}$, as indicated by improved agreement values.

\section{Discussion}

Theoretically, the optimal packing of iron atoms around the central carbon atoms in the monoclinic structure is simple trigonal prisms (Hirotsu \& Nagakura, 1972; Dirand \& Afqir, 1983). In the Pī space group, both the iron and carbon atoms are allowed additional positional freedom during the refinement. This provided a better fit of the experimental diffractogram 
and suggests that the iron atoms in the unit cell are arranged in three different types of distorted trigonal prisms and an octahedron around the central carbon atoms, compare Figure 5 .
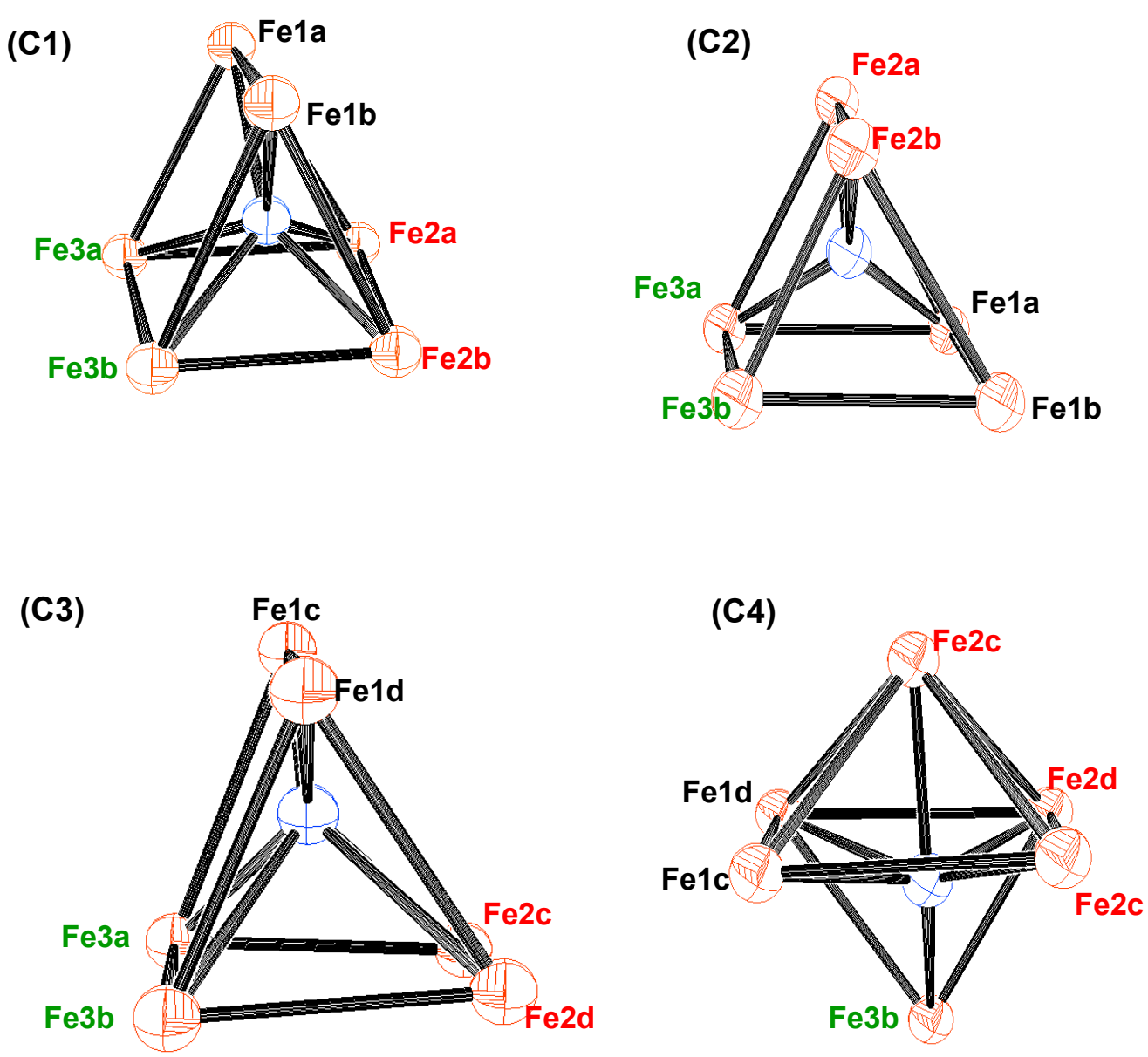

Figure 5 The three different types of distorted trigonal prisms of iron and octahedron (shaded around the central carbon atoms (no shading) for all four carbon atoms in the unit cell.

The PDF approach was used to refine the nano-crystalline structure of $\chi$-HC. From the agreement between the Rietveld and PDF refinements, it can be deduced that the bulk structure probably extends to the surface of the powder particles. Differences in relative abundances between Rietveld and PDF quantitative refinements usually indicate local structural disorder (Egami et al, 2003). The distortions of the trigonal prisms are thus not due to local disorder, but possibly due to mobility of carbon in the bulk structure. The relative atomic coordinates as fractions of the unit cell of $\chi$-HC are listed in Table 2 . The values $\Delta \mathrm{x}, \Delta \mathrm{y}$ and $\Delta \mathrm{z}$ indicate the deviations in the relative atomic coordinates of the atoms from those in the monoclinic structure $(\mathrm{C} 2 / \mathrm{c})$. These relative atomic coordinate displacement values suggest that the carbon atoms could be mobile in the $\chi$-HC crystal structure. 
Table 2 Relative atomic coordinates of $\chi$ - $\mathrm{HC}$, and deviations from the theoretical $\mathrm{C} 2 / \mathrm{c}$ optimal packing.

\begin{tabular}{lllllll}
\hline Atom & $\mathbf{x}$ & $\Delta \mathbf{x}$ & $\mathbf{y}$ & \multicolumn{1}{l}{$\mathbf{y}$} & $\mathbf{z}$ & $\Delta \mathbf{z}$ \\
\hline Fe1a & $0.3532(1)$ & -0.007 & $0.3269(1)$ & 0.005 & $0.4281(1)$ & -0.009 \\
Fe1b & $0.6512(1)$ & 0.002 & $0.8179(2)$ & 0.014 & $0.0856(3)$ & -0.005 \\
Fe1c & $0.1539(1)$ & 0.000 & $0.3353(3)$ & -0.003 & $0.0898(3)$ & -0.009 \\
Fe1d & $0.8370(1)$ & 0.010 & $0.8447(2)$ & -0.013 & $0.4179(2)$ & 0.002 \\
Fe2a & $0.4558(1)$ & 0.008 & $0.8387(3)$ & -0.003 & $0.2939(3)$ & 0.012 \\
Fe2b & $0.5275(1)$ & 0.009 & $0.3505(3)$ & -0.015 & $0.1979(3)$ & -0.004 \\
Fe2c & $0.0378(1)$ & -0.002 & $0.8288(3)$ & 0.007 & $0.1820(2)$ & 0.012 \\
Fe2d & $0.9677(1)$ & -0.004 & $0.3358(3)$ & 0.000 & $0.3087(3)$ & -0.003 \\
Fe3a & $0.2523(1)$ & -0.002 & $0.8270(3)$ & 0.000 & $0.2488(3)$ & 0.001 \\
Fe3b & $0.7462(1)$ & 0.004 & $0.3291(3)$ & -0.003 & $0.2489(3)$ & 0.001 \\
C1 & $0.3641(5)$ & 0.006 & $0.552(1)$ & -0.016 & $0.074(1)$ & 0.019 \\
C2 & $0.6109(5)$ & 0.019 & $0.010(1)$ & 0.026 & $0.358(1)$ & 0.049 \\
C3 & $0.1357(2)$ & -0.006 & $0.545(1)$ & -0.009 & $0.434(1)$ & -0.027 \\
C4 & $0.887(1)$ & -0.017 & $0.107(1)$ & -0.071 & $0.067(1)$ & 0.026 \\
\hline
\end{tabular}

To summarise, high-resolution synchrotron X-ray diffraction data was obtained on beam line ID31 at the ESRF, Grenoble, and confirmed the pseudo-monoclinic structure of $\chi$-HC. In addition to confirmation of the true crystal structure of $\chi-\mathrm{HC}$, the presence of 1-2\% weight iron was detected in the sample using synchrotron X-rays, which was not visible using laboratory XRD. Using the PDF approach it was also possible to refine the nano-crystalline structure of $\chi-\mathrm{HC}$, which proved the triclinic structure to be prevalent in the unloaded catalysts. It is also clear from comparing Rietveld and PDF quantative phase analyses that the bulk $\chi$-HC structure extends to the surface of the $\chi$-HC particles. The iron atoms are located in three distorted prismatic trigonal and one octahedral arrangement around the central carbon atoms, an arrangement which is significantly different from the monoclinic structure of $\chi-\mathrm{HC}$ as described and reported previously.

Acknowledgements We gratefully acknowledge Sasol Technology R\&D Pty Ltd, South Africa, for funding, and acknowledge the ESRF (Grenoble, France) for provision of beam time on the high-resolution powder diffraction beam line ID31. 


\section{References}

1 Coelho, A. A. (2000). J. Appl. Cryst., 33, 899-908.

2 Dirand, M., Afqir, L. (1983). Acta Metall. 31, 1089-1107.

3 Dry, M. E., (1990). Catalysis Letters 7, 241-252.

4 du Plessis, H. E., de Villliers, J.P.R., Kruger, G.J.,. (2007). Z. Kristallogr., 211-217.

5 Faraoun, H.I., Zhang, Y.D., Esling, C., Aourag, H., (2006), J. Appl. Phys. 99, 093508-1-8.

6 Farrow, C.L., Juhas, P., Liu, J.W., Bryndin, D., Bozin, E.S., Bloch, J., Proffen, T., Billinge, S.J.L., (2007). J. Phys.: Condens. Matter. 19, 335219.

7 Hagg, G., (1931). Physic. Chem. B12, 33-56.

8 Herranz, T., Rojas, S., Perez-Alonso, F.J., Ojeda, M., Terreros, P. , Fierro, J.L.G., (2006). J. of

Catalysis, 243, 199-211.

9 Hirotsu, Y., Nagakura, S. (1972). Acta Metallurgica 20, 645-655.

10 Königer, A., Hammerl, C., Zeitler, M., Rauschenbach, (1997). Phys. Rev. B 55, 13, 8143 - 8147.

11 Mansker, L. D. (1999), PhD thesis, The University of New Mexico, New Mexico.

12 Meinhardt, D., Krisement, O. (1962). Arch. Eisenhuettenwes. 33, 493 - 499

13 Niemantsverdriet, J. W., (1980). J. Phys. Chem. 84, 3363-3370.

14 Pérez-Alonso, F.J., Herranz, T., Rojas, S., Ojeda, M., López Granados, M., Terreros, P., Fierro,

J.L.G., Gracia, M., Gancedo, J.R., (2007). Green Chem., 9, 663-670.

15 Qiu, X., Thompson, J. W., Billinge, S. J. L., (2004). J. Appl. Crystal. 37, 678-678.

16 Retief, J. J., (1999). Powder Diffraction 14, 130-132.

17 Riedel, T., Schulz, H., (2003) Topics in Catalysis, 26, 1-4, 41-54.

18 Sarkar, A., Seth, D., Dozier, A.K., Neathery, J.K., Hamdeh, H.H., Davis, B.H., (2007). Catal. Lett., 117, 1-17.

19 Schneider, A., Inden, G., (2007). Computer Coupling of Phase Diagrams and Thermochemistry, 31, 141-147.

20 Senateur, J. P., (1962). Comptes Rendus Acad. Sci. 255, 1615-1616.

21 Snyder, R.L., Bunge, H.J., Fiala, J., (1999). Editors. Microstructure Analysis from Diffraction, 94124.

22 Steynberg, P.J., van den Berg, J.A., Janse van Rensburg, W., (2008). J. Phys.: Condens. Matter 20, 064238 .

23 Swanson, H.E., Fuyat, R.K., Ugrinic, G.M., (1955), Standard X-ray Diffraction Powder Patterns. Natl. Bur. Stand. (U.S.), Circ. 539, IV, 75.

24 Young, R. A. (1995). Editor. The Rietveld method. Oxford: University Press. 